\title{
Shock Waves in Magnetogasodynamic Turbulence
}

\author{
S. A. Kaplan
}

\author{
Astronomical Observatory, Lvov, U.S.S.R.
}

$\mathrm{T}$ HE spectral theory of isotropic magnetogasodynamic turbulence proposed by the author some years ago $^{1,2}$ gives the following expression for the spectral functions of kinetic and magnetic energies.

(a) Stationary case:

$$
\begin{gathered}
F(k) \sim \frac{v_{k}{ }^{2}}{2 k} \sim k^{1-2 \alpha}, \quad G(k) \sim \frac{H_{k}^{2}}{8 \pi \rho \kappa} \sim k^{1-2 \alpha}, \\
\alpha=1-\frac{3 \eta_{f}+\eta_{g}}{4 \zeta_{f}-\zeta_{g}}+\left\{1+\frac{1}{2 \zeta_{f}-\zeta_{g}}+\frac{\eta_{f}+\eta_{g}}{16}\left(\frac{9}{\zeta_{f}-\zeta_{g}}\right)^{2}\right\}^{\frac{1}{2}} .
\end{gathered}
$$

(b) Quasi-stationary case :

$$
\begin{aligned}
& F(k) \sim \frac{v_{k}{ }^{2}}{2 k} \sim k^{1-2 \alpha}, \quad G(k) \sim \frac{H_{k}{ }^{2}}{8 \pi \rho \kappa} \sim k^{1-\alpha}, \\
& \alpha=1-\frac{3}{4} \frac{\eta_{f}}{\zeta_{f}}+\left\{1+\frac{1}{2} \frac{\eta_{f}}{\zeta_{f}}+\frac{9}{16}\left(\frac{\eta_{f}}{\zeta_{f}}\right)^{2}\right\}^{\frac{1}{2}},
\end{aligned}
$$

where $k=2 \pi / \ell$ is the wave number of eddies or motions of scale $\ell, v_{k}$ and $H_{k}$ the velocity and magnetic field of motions in these scales, and $F(k)$ and $G(k)$ the spectral functions of the kinetic and magnetic energies, respectively. In other words, $F(k) d k$ and $G(k) d k$ are the quantities of the kinetic and magnetic energies per unit mass contained in the motions with wave numbers in the interval from $k$ to $k+d k ; \rho$ the gas density, $\zeta$ and $\zeta_{g}$ the dimensionless quantities describing the full dissipation of the kinetic energy and the increase of the magnetic energy in shock waves. Therefore, $\zeta_{f}-\zeta_{g}$ describes the dissipation of the kinetic energy of shock waves into thermal energy. $\eta_{f}$ and $\eta_{g}$ are also dimensionless quantities describing the transfer of the kinetic energy and, respectively, the magnetic energy from big scale to lesser motions (the inertial members of the magnetogasodynamic equations).

If the dissipation of energy in shock waves in magnetogasodynamic turbulence is unimportant, we have $\eta_{f}+\eta_{g}>\zeta_{f}-\zeta_{g}$ or $\eta_{f} \gg \zeta_{f}$ and therefore $\alpha \rightarrow 4 / 3$. We obtain then Kolmogorov's spectra for the kinetic and the magnetic energies:

$F(k) \sim k^{-5 / 3}, \quad G(k) \sim k^{-5 / 3}$ (stationary case); $F(k) \sim k^{-5 / 3}, \quad G(k) \sim k^{-1 / 3}$ (quasi-stationary case).

On the contrary, if the dissipation of energy in shock waves is larger than the transfer of energy between the motions of different scales, we have $\eta_{f}+\eta_{g} \ll \zeta_{f}-\zeta_{g}$ or $\eta_{f} \ll \zeta_{f}$. Therefore $\alpha \rightarrow 2$, and we obtain

$$
\begin{array}{ll}
F(k) \sim k^{-3}, & G(k) \sim k^{-3} \text { (stationary case); } \\
F(k) \sim k^{-3}, & G(k) \sim k^{-1} \text { (quasi-stationary case). }
\end{array}
$$

I S. A. Kaplan, J. Exptl. i Theoret. Phys. 27, N6, 699, (1954)

2 S. A. Kaplan, "Theory of isotropic magneto-gasodynamic turbulence," Symposium on Hydromagnetics in Stockholm (August, 1956).
From this it follows that in magnetogasodynamic turbulence, in which shock waves occur, the dependence of the velocities of eddies upon the scale $\ell$ of motions is as follows:

$$
\frac{1}{3} \leqslant \frac{d \ln v_{e}}{d \ln \ell} \leqslant \frac{1}{2} .
$$

The magnetic fields $H_{e}$ depend upon the scale of field fluctuations in such a way that

$$
\begin{aligned}
\frac{1}{3} & \leqslant \frac{d \ln H_{e}}{d \ln \ell} \leqslant \frac{1}{2} \quad \text { (stationary case) } \\
-\frac{1}{3} & \leqslant \frac{d \ln H_{e}}{d \ln \ell} \leqslant 0 \quad \text { (quasi-stationary case). }
\end{aligned}
$$

The quasi-stationary case of magnetogasodynamic turbulence corresponds to Batchelor's picture of magnetohydrodynamic turbulence, in which the magnetic energy is chiefly contained in small scale motions.

The increasing role of energy dissipation in the shock waves results in an increase of the index of the degree of spectral functions.

The author has tried to find the dependence of the velocity and the magnetic field upon the scale of motions in the interstellar space. An analysis of the radial velocities of interstellar gas clouds shows that, up to $80 \mathrm{pc}{ }^{3}$

$$
d \ln v_{e} / d \ln \ell=0.36,
$$

which is in good accordance with Eq. (7). Respectively, an analysis of the observational data of interstellar polarization shows (with great uncertainty) that

$$
d \ln H_{e} / d \ln \ell \approx 0.12 \text {. }
$$

This value does not agree with Eqs. (8) or (9), but it is necessary to keep in mind that in this case the observational dependence does not coincide with the actual dependence, because we do not consider a single value of $H_{e}$, but an average over some hundred parsecs along the line of sight. If this effect is duly taken into account, we get

$$
d \ln H_{e} / d \ln \ell \approx-0.38,
$$

which also does not agree with Eqs. (8) or (9). In view of the uncertainty of this value this difference does not seem to be very serious. It is interesting to note that the observational data are favorable in the case of quasistationary turbulence.

Both Eqs. (10) and (12) are nearer to the lower limits of the indices of the degree of turbulent spectrum; therefore, the dissipation of shock wave energy in interstellar turbulence is unimportant.

\footnotetext{
${ }^{3}$ S. A. Kaplan, Astron. Zhur. 32, N3, 255, (1955).
} 